\title{
Immunofibroblasts are the cornerstone of TLS formation in pSS
}

\section{ce \\ proliferation \\ of immuno- \\ fibroblasts \\ preceded the \\ infiltration of \\ lymphocytes \\ into tissues}

\author{
Aggregates of lymphocytes and \\ stromal cells known as tertiary \\ lymphoid structures (TLSs) form \\ in peripheral tissues and contribute \\ to autoantibody production and \\ pathology in autoimmune \\ diseases such as primary Sjögren \\ syndrome (pSS). Insight into the \\ mechanism by which TLSs form \\ in pSS is raising hope that this \\ process could one day be targeted \\ therapeutically. \\ "We believed that this process \\ was driven, within the TLS, by the \\ presence of 'immunofibroblasts', \\ a population of non-haematopoietic \\ stromal cells that are capable of \\ supporting both B and T lymphocyte \\ survival, migration, activation \\ and proliferation and that have \\ similar functional properties to \\ the stromal fibroblasts described \\ in secondary lymphoid organs," \\ explains co-corresponding author \\ Francesca Barone.
}

\section{On the basis of this} belief, Barone and colleagues examined TLSs from patients with pSS, which contained podoplanin-positive fibroblasts that expressed molecules related to either lymphocyte survival within TLSs or to TLS organization. In vitro, these 'immunofibroblasts' proliferated and upregulated cell adhesion molecules in response to IL-22 and IL-13, respectively.

Using a viral infection model of pSS in various types of knockout mice, the researchers unravelled the precise mechanisms by which these immunofibroblasts participate in TLS formation. Activation and proliferation of immunofibroblasts preceded the infiltration of lymphocytes into tissues, was indispensable for TLS formation and was controlled by both IL- 13 and IL-22. "Interestingly these cytokines are very different from the signalling

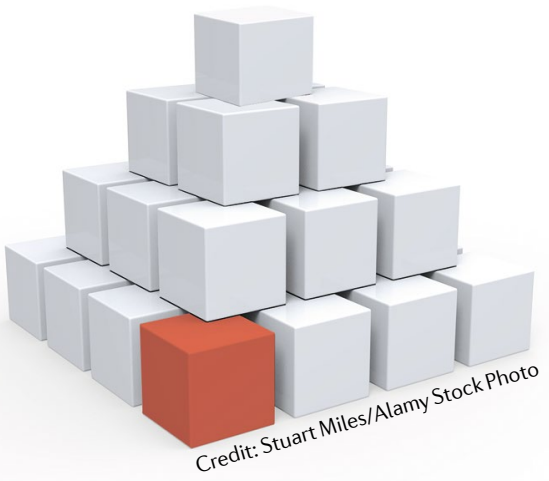

programme that drives the formation of secondary lymphoid organs," notes Barone.

"Blocking these cytokines, or eliminating the immunofibroblasts in vivo, was sufficient to disrupt TLSs," states co-corresponding author Mark Coles. "This is particularly important because drugs targeting these two different cytokines have already been developed for other inflammatory conditions, thus can be repurposed for TLS-associated pathologies."

Joanna Collison

ORIGINAL ARTICLE Nayar, S. et al.

Immunofibroblasts are pivotal drivers of tertiary lymphoid structure formation and local pathology. Proc. Natl Acad. Sci. USA 116, 13490-13497 (2019)

\section{Gene therapy counteracts bone loss in osteoporosis}

Silencing expression of the adaptor protein Schnurri-3 (SHN3) via bonetargeting adeno-associated virus (AAV)-mediated gene therapy could be an attractive approach to treat osteoporosis by promoting bone formation, according to new research published in Nature Communications.

Previous work by the researchers had identified SHN3 as a potent suppressor of bone formation. The current paper highlights the therapeutic effects of silencing SHN3 in a mouse model of osteoporosis. The researchers used a recombinant AAV (rAAV) vector carrying an artificial microRNA (amiR) that targets Shn3 (amiR-Shn3). "This technology can confer gene silencing to osteoblast lineage cells residing on the bone surface with a single administration to dramatically alter bone physiology," explains corresponding author Jae-Hyuck Shim.
Shim and colleagues showed that administration of rAAV9-amiR-Shn3 either systemically or directly to the knee joints of mice reduced Shn3 expression in osteoblast-lineage cells and increased osteoblast activity and trabecular bone mass, compared with a control amiR delivered by rAAV9. Furthermore, a single systemic administration of rAAV9-amiR-Shn3 after the onset of ovariectomy-induced osteoporosis reversed trabecular bone loss and enhanced femur strength and stiffness in mice. "These findings suggest that rAAV9-mediated silencing of Shn3 improves clinically meaningful endpoints," reports Shim. Notably, systemic delivery of rAAV9-amiR-Shn3 did not affect osteoclast function in vivo.

Observing that rAAV9 can extensively transduce tissues other than bone, the researchers grafted a bone-targeting peptide motif onto the AAV9 capsid

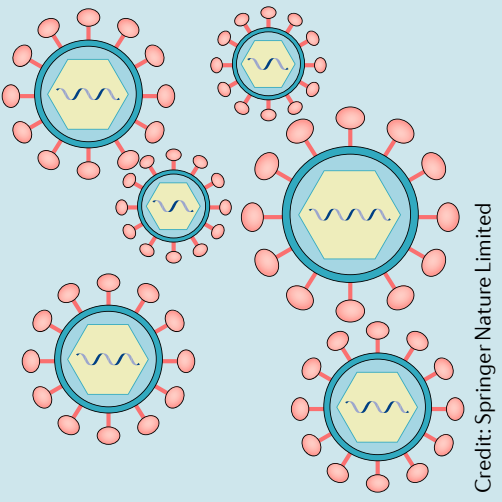

scaffold to produce a novel bone-specific capsid of rAAV9 that reduced transgene expression in non-bone peripheral organs, including liver, heart and muscle.

"Systemic delivery of amiR-Shn3 via bone-targeting AAV9 has clinical utility for counteracting bone loss during osteoporosis," concludes Shim. "However, much more work needs to be done to establish the safety and efficacy of this therapeutic approach."

Sarah Onuora

ORIGINAL ARTICLE Yang, Y.-S. et al. Bone-targeting AAV-mediated silencing of Schnurri-3 prevents bone loss in osteoporosis. Nat. Commun. 10, 2958 (2019) 